\title{
DISTRIBUCIÓN ESPACIAL DE ALGUNAS PROPIEDADES FISICAS DEL SUELO EN UN TRANSECTO DE LA GRANJA TUNGUAVITA, PAIPA
}

\section{SPATIAL DISTRIBUTION OF SOME FISICAL SOIL PROPERTIES IN A TRANSECT OF THE TUNGUAVITA FARM, PAIPA}

\author{
Fanny Liliana Monroy-Rodríguez ${ }^{1}$, Javier Giovanni Álvarez-Herrera², Óscar Humberto Alvarado-Sanabria ${ }^{3}$
}

\begin{abstract}
${ }^{1}$ Ingeniera Agrónoma, Facultad de Ciencias Agropecuarias. Universidad Pedagógica y Tecnológica de Colombia, Av. Central del Norte, Tunja, Colombia, e-mail: fannymonroy@fedearroz.com.co; ${ }^{2}$ Ingeniero Agrícola, M.Sc., Ph.D., Profesor asistente, grupo de investigaciones agrícolas, Facultad de Ciencias Agropecuarias. Universidad Pedagógica y Tecnológica de Colombia, Av. Central del Norte, Tunja, Colombia, e-mail: javier.alvarez@uptc.edu.co; ${ }^{3}$ Ingeniero Agrónomo, c.M.Sc. Fisiología Vegetal, grupo de investigaciones agrícolas. Universidad Pedagógica y Tecnológica de Colombia, Av. Central del Norte, Tunja, Colombia, e-mail: ohalvarados@unal.edu.co
\end{abstract}

Rev. U.D.C.A Act. \& Div. Cient. 20(1): 91-100, Enero-Junio, 2017

\section{RESUMEN}

El uso de técnicas geoestadísticas permite conocer la variabilidad de las propiedades de los suelos y facilita la interpretación, la predicción y la toma de decisiones. Con el fin de establecer el impacto del manejo que se le ha dado a la granja Tunguavita, se realizó un transecto, en el cual, se tomaron 85 puntos de muestreo, que se encontraban en un zona de bosque, dedicada a pastos y a explotación equina. Se determinaron las relaciones de masa-volumen, las humedades en profundidad y las resistencias a la penetración a dos profundidades. La aireación fue la relación de masavolumen que presentó el mayor coeficiente de variación, lo que implica un manejo diferente a lo largo del transecto evaluado. La densidad real y la porosidad tienen una baja dispersión en el espacio. Las densidades aparente y real, la porosidad, la aireación y la relación de vacíos tuvieron un rango superior a $1000 \mathrm{~m}$. Los muestreos para la resistencia a la penetración y las humedades medidas con TDR, se deberían hacer cada $600 \mathrm{~m}$. La resistencia a la penetración mostró ser inversamente proporcional al contenido de humedad en el suelo. Las variables medidas se correlacionan en dos grupos: el primero, asocia a las densidades con las humedades medidas a diferentes profundidades $\mathrm{y}$, el segundo grupo, relaciona la resistencia a la penetración a $15 \mathrm{~cm}$ con la medida a $30 \mathrm{~cm}$. La resistencia a la penetración es un buen indicador de la compactación del suelo y del manejo agropecuario que se da en la granja Tunguavita.

Palabras clave: Geoestadística, semivariograma, kriging, compactación de suelo, variabilidad

\section{SUMMARY}

The use ofgeostatistical techniques allows to find the variability of soil properties and also facilitates interpretation, prediction and decision making. Therefore, in order to establish the impact of management that has been given to the farm Tunguavita, a transect was conducted in which 85 sample points were taken, located found in a wooded area, an area devoted to pasture, and an area of equine exploitation. Volume mass ratios, humidities in depth and resistance to penetration at two depths was determined. Aeration was the mass-volume ratio that had the highest variation coefficient, which implies the different management practices along the evaluated transect. The particle density and porosity have a low dispersion in space. Bulk and particle densities, porosity, aeration and voids ratio presented a range higher than $1000 \mathrm{~m}$. Therefore, sampling for penetration resistance and moisture with TDR measurements should be done each $600 \mathrm{~m}$. Resistance to penetration showed to be inversely proportional to the moisture content in the soil. The measured variables are correlated in two groups, the first one associated densities with the humidities, measured at different depths, and the second group related to penetration resistance at $15 \mathrm{~cm}$ with measurement at $30 \mathrm{~cm}$. The penetration resistance is a good indicator of soil compaction and the agricultural management that occurs in the Tunguavita farm.

Key words: Geostatisttca, semivariogram, kriging, soil compaction, variability. 


\section{INTRODUCCIÓN}

Los métodos geoestadísticos han sido una herramienta ampliamente utilizada por los científicos del suelo en las dos últimas décadas, ya que permiten estudiar la variabilidad y la estructura de la variación de las propiedades físicas, químicas y morfológicas (Sun et al. 2003), de tal forma que el conocimiento de la variabilidad espacial de las propiedades de los suelos se ha convertido en esencial, para realizar un manejo adecuado de los suelos (Mulla, 2012).

Las propiedades físicas de los suelos han sido estudiadas geoestadísticamente por diferentes autores, quienes afirman que estas propiedades varían de un lugar a otro, dependiendo del manejo dado al suelo y sus propias características (Mulla, 2012). La variabilidad espacial de las propiedades físicas del suelo dentro de los campos agrícolas es inherente a la naturaleza geológica de la formación de suelo, pero, en algunos casos, la variabilidad puede ser inducida por la labranza y otras prácticas de manejo. Estos factores interactúan unos con otros, a través de escalas espaciales y temporales y se modificarán una vez más, a nivel local, por los procesos de erosión y deposición (lqbal et al. 2005).

Por el desconocimiento de las propiedades físicas del suelo y su relación con la compactación, se ha dificultado el manejo de factores importantes para la explotación de cultivos y la adecuada utilización del recurso suelo, por lo que se hizo necesario el estudio espacial de algunas propiedades y sus variaciones dependientes de la naturaleza geológica o del manejo y la mecanización que se le dé al suelo, la cual, es la provocada por cambios que ocurren en el tiempo y que son generados, en gran medida, por el manejo, debido al tipo de uso del suelo (Tasumi \& Kimura, 2013). Del mismo modo, otra fuente de variación es atribuida a que la variabilidad de las propiedades del suelos puede ser inducida por la labranza y otras prácticas de manejo, como la explotación animal (Zucco et al. 2014).

Por lo anterior, este artículo se centró en determinar la distribución espacial de algunas propiedades del suelo y su relación con la compactación, en un transecto de la granja Tunguavita del municipio de Paipa, que servirá de ayuda para el manejo adecuado del agua y de los suelos, de una gran área de la zona del alto Chicamocha, la cual, presenta condiciones similares.

\section{MATERIALES Y MÉTODOS}

Localización. El estudio, se desarrolló en la granja Tunguavita, ubicada en el municipio de Paipa, a una altitud de $2.470 \mathrm{msnm}$; con latitud $05^{\circ} 44^{`} 27^{\prime \prime}$ Norte y longitud $73^{\circ} 07^{`} 15^{\prime \prime}$ Oeste; la temperatura media es de $14,1^{\circ} \mathrm{C}$; precipitación media anual de 966mm y la humedad relativa media de $75 \%$; la granja tiene una extensión total de 134ha.

Procedimiento. Se realizó un estudio de altimetría con la ayuda del sistema de posicionamiento global (GPS), con el fin de establecer mapas de la distribución de las variables en la zona de estudio, en zonas con posibles problemas de compactación e inundaciones frecuentes. Para determinar la variabilidad espacial de las propiedades en estudio, se realizó un transecto de 85 puntos, considerando un efecto anisotrópico igual a cero, con la siguiente distribución: distancias de $10 \mathrm{~m}$ entre cada muestra. Se tomaron muestras, a una profundidad de $15 \mathrm{~cm}$ en cada punto y para cada variable. Se hizo un análisis estadístico descriptivo y se formalizó una prueba de normalidad, además un estudio geoestadístico, en el cual, se realizaron semivariogramas, interpolación por kriging puntual y validación cruzada, para determinar la variabilidad espacial de los parámetros.

La dependencia espacial de los atributos, se calculó usando la teoría de las variables regionalizadas, en la cual, un grupo de datos es ajustado a un modelo de semivariograma teórico $\gamma(\mathrm{h})$, definido de la siguiente forma:

$$
\gamma(h)=\frac{1}{2 N(h)} \sum_{i=1}^{N(h)}[Z(X i)-Z(X i+h)]^{2}
$$

Donde, $\gamma(h)=$ Semivarianza, $N(h)=$ Número de parejas de puntos separados a una distancia $\mathrm{h} ; \mathrm{Z}(\mathrm{Xi})=$ Valor del atributo en el lugar $\mathrm{Xi}$ y $Z(\mathrm{Xi}+\mathrm{h})=$ Valor del atributo a una distancia $\mathrm{h}$ del lugar $\mathrm{Xi}+\mathrm{h}$.

De esta manera, se determinó la autocorrelación espacial de las variables y la correlación entre las variables en un área determinada. En general, Garzón et al. (2010) menciona que si los puntos cercanos se parecen mucho entre sí, se dice que existe una autocorrelación espacial positiva, si por el contrario, los puntos cercanos difieren mucho, no existe autocorrelación, es decir, se presenta independencia espacial de la variable. De acuerdo al ajuste de los semivariogramas, se obtuvieron modelos exponenciales, gaussianos y lineales.

Variables de respuesta. En cada punto, se determinó el porcentaje de arena, limo y arcilla, por el método de Bouyoucos. Se obtuvo la densidad aparente, el contenido de humedad gravimétrica, volumétrica y saturación, así como la porosidad, la aireación y la relación de vacíos, por el método del cilindro biselado, mediante el muestreador Bulman modelo 0200 (Beavercreek, Ohio) y se determinó la densidad real, por el método del picnómetro (USDA, 2004). La compactación del suelo, se midió con el equipo FieldScout SC-900, mediante datos de resistencia a la penetración y se determinó la humedad a diferentes 
profundidades, con un Time Domain Reflectometer (TDR), marca FieldScout TDR 100.

Análisis de datos. Se realizó, inicialmente, un análisis estadístico descriptivo, con el fin de determinar los parámetros básicos y las tendencias de los datos. Así mismo, se efectuó una prueba de normalidad, mediante el test de bondad de ajuste de Kolmogorov Smirnov. Se hizo un análisis geoestadístico, que incluyó ajustes de datos a modelos teóricos de semivariogramas, en donde se determinó el efecto pepita $\left(\mathrm{C}_{0}\right)$; la meseta $\left(\mathrm{C}_{0}+\mathrm{C}\right)$; el rango y el grado de dependencia espacial (GDE), mediante la relación $\mathrm{C} /\left(\mathrm{C}_{0}+\mathrm{C}\right)$. Los datos, se validaron mediante el modelo de ajuste de Krigingy, posteriormente, se realizó la validación cruzada; para la realización de los semivariogramas, se usó el software Gs+ v5.1. Del mismo modo, se practicó un análisis multivariado, en el cual, se llevó a cabo un análisis de correlación lineal simple y múltiple, por medio de la correlación de Pearson y del análisis de componentes principales, respectivamente, así como del efecto de comunalidad, mediante el software Sas v.8.1e (Cary N.C.).

\section{RESULTADOS Y DISCUSIÓN}

Análisis descriptivo. Las propiedades analizadas mostraron un comportamiento normal, según la prueba de Kolmogorov-Smirnov, a excepción de la humedad medida por el TDR, a $4 \mathrm{~cm}$, la humedad gravimétrica y la resistencia a la penetración (Tabla 1). Según Glendell et al. (2014), la normalidad no es un requisito obligatorio para el análisis de datos geoestadísticos; no obstante, si los datos son normales permiten una comparación más confiable de los parámetros de los modelos y brinda estimaciones del Kriging con mejor ajuste.

Densidad aparente (Da). De acuerdo con la prueba de normalidad de Shapiro-Wilk, la densidad aparente tuvo una distribución normal, con una media y mediana cercanas. Esto lo confirma la curtosis, con un valor cercano a cero y con un comportamiento platicúrtico. El valor promedio de la densidad aparente fue inferior en un $65 \%$ al reportado por Guatibonza et al. (2009), quienes obtienen el mismo comportamiento normal, pero con una densidad aparente, superior de $1,27 \mathrm{gcm}^{-3}$. Esta diferencia, se puede deber a que ellos tomaron muestras en una época seca, mientras que, para este estudio, los valores se tomaron en enero de

Tabla 1. Estadística descriptiva de las variables medidas.

\begin{tabular}{|c|c|c|c|c|c|c|c|c|}
\hline Parámetro & Media & Mediana & Mínimo & Máximo & Asimetría & Curtosis & CV & N \\
\hline HG (\%) & 57,90 & 46,46 & 9,74 & 132,22 & 0,79 & 0,15 & 45,88 & $*$ \\
\hline HV (\%) & 42,51 & 42,47 & 13,90 & 62,45 & $-0,62$ & 0,77 & 22,29 & $\mathrm{~ns}$ \\
\hline HS (\%) & 69,16 & 71,27 & 32,45 & 86,62 & $-1,04$ & 0,93 & 18,37 & $\mathrm{~ns}$ \\
\hline Poros (\%) & 61,48 & 60,77 & 35,14 & 79,95 & $-0,22$ & $-0,09$ & 14,17 & $\mathrm{~ns}$ \\
\hline A (\%) & 18,97 & 17,93 & 6,71 & 43,04 & 0,79 & 0,34 & 42,60 & $\mathrm{~ns}$ \\
\hline Vacíos & 1,74 & 1,55 & 0,54 & 3,99 & 0,92 & 0,75 & 38,55 & $\mathrm{~ns}$ \\
\hline Da ( $\left.\mathrm{g} \mathrm{cm}^{-3}\right)$ & 0,83 & 0,85 & 0,39 & 1,43 & 0,28 & $-0,28$ & 27,76 & $\mathrm{~ns}$ \\
\hline Dr $\left(\mathrm{g} \mathrm{cm}^{-3}\right)$ & 2,12 & 2,13 & 1,61 & 2,46 & $-0,41$ & 0,05 & 7,94 & $\mathrm{~ns}$ \\
\hline H4 (\%) & 94,07 & 98,00 & 59,70 & 99,60 & $-2,49$ & 6,27 & 8,88 & $*$ \\
\hline H8 (\%) & 80,08 & 81,20 & 44,40 & 99,00 & $-0,60$ & 0,51 & 15,34 & $\mathrm{~ns}$ \\
\hline H12 (\%) & 57,68 & 55,70 & 29,30 & 99,00 & 1,36 & 2,60 & 24,89 & $\mathrm{~ns}$ \\
\hline H20 (\%) & 42,44 & 41,20 & 20,40 & 85,70 & 1,67 & 4,18 & 27,87 & $\mathrm{~ns}$ \\
\hline RP1 (MPa) & 1,33 & 1,17 & 0,30 & 3,70 & 1,53 & 3,12 & 48,4 & $*$ \\
\hline RP2 (MPa) & 1,73 & 1,62 & 0,78 & 3,70 & 1,38 & 2,42 & 35,68 & $*$ \\
\hline
\end{tabular}

HG: humedad gravimétrica; HV: Humedad volumétrica; HS: Humedad a saturación; A: Aireación; Da: Densidad aparente en seco; DAH: densidad aparente en húmedo; Dr: Densidad real; H4: humedad a 4cm por TDR; H8: humedad a $8 \mathrm{~cm}$ por TDR; H12: humedad a $12 \mathrm{~cm}$ por TDR; H20: humedad a $20 \mathrm{~cm}$ por TDR. RP1: resistencia a la penetración a $15 \mathrm{~cm}$; RP2: resistencia a la penetración a $30 \mathrm{~cm}$; N: Normalidad de Kolmogorov - Smirnov; ns: no significativo; *: significativo para normalidad $(P<0,01)$. 
2012, justo después de uno de los años más lluviosos en la zona (Figura 1), por lo que se puede inferir que la Da cambia con la humedad. Al analizar el coeficiente de variación (CV), presentó un valor de $27,7 \%$, considerado alto, de acuerdo a la escala usada por Garzón et al. (2010). Esta situación puede ocurrir, debido a que el transecto de muestreo correspondía a zonas de bosque, zonas de pastoreo y hacia la parte norte de la zona de muestreo, se tenía un lugar de alta compactación dedicado a la explotación equina, mientras el estudio de Guatibonza et al. (2009), se llevó a cabo en forma de malla, con muestreos más cercanos entre sí y presentó un CV más bajo, con un valor de 16,6\%.

En cuanto a la densidad real (Dr), los datos tuvieron un comportamiento normal, lo que lo confirma la similitud entre media y mediana. La curva de estos datos presentó asimetría y un comportamiento platicúrtico, similar a lo reportado por Cucunuba-Melo et al. (2011), quienes encontraron una media y mediana similares, con valores de 2,53 y 2,50, respectivamente y una curtosis de 0,01 . El CV encontrado para la densidad real es bajo (Tabla 1), debido a que la densidad real esta relacionada a la génesis del suelo y hace que presente poca variación, similar a lo obtenido por Guatibonza et al. (2009) y Cucunuba-Melo et al. (2011).

La humedad gravimétrica presentó un comportamiento anormal, en donde la media es mayor que la mediana. La distribución de los datos mostró sesgo hacia la izquierda y un comportamiento platicúrtico. Por otro lado, la humedad volumétrica y de saturación presentó un comportamiento normal, con una media y mediana similar, una asimetría negativa y una curva platicúrtica. Allaire et al. (2012) mencionan que el comportamiento normal de la humedad volumétrica ha sido reportado por otros autores, aunque ellos obtuvieron un menor coeficiente de variación, con un valor de $14 \%$, mientras que, en este estudio, los valores de $\mathrm{CV}$, oscilaron entre 18 y 45\%, lo que pudo ocurrir, debido a la extensión del transecto medido, ya que se realizó en zonas con alta variabilidad, lo que aumentó el CV.

La porosidad tuvo un comportamiento normal, caracterizado por una curva platicúrtica y con una asimetría negativa; esta normalidad ha sido reportada por otros autores (CucunubaMelo et al. 2011; Dos Santos et al. 2012), quienes también relacionan este comportamiento normal con el de la densidad aparente y la densidad real, ya que esta variable tiene fuerte relación con las densidades. Según Lipiec \& Hatano (2003), un valor promedio, como el encontrado de $61 \%$, implica un suelo con una buena aireación y almacenamiento de agua. Del mismo modo, el CV hallado para la porosidad es bajo (Tabla 1) y es similar al que Guatibonza et al. (2009) reportaron para la misma zona, en época seca.

La aireación del suelo presentó un comportamiento normal, con una media y mediana similar y con una curva platicúrtica y asimetría positiva; no obstante, presentó mayor variación que otras variables, con un CV del $42 \%$, lo que concuerda con Hernández et al. (2007), quienes encontraron coeficientes de

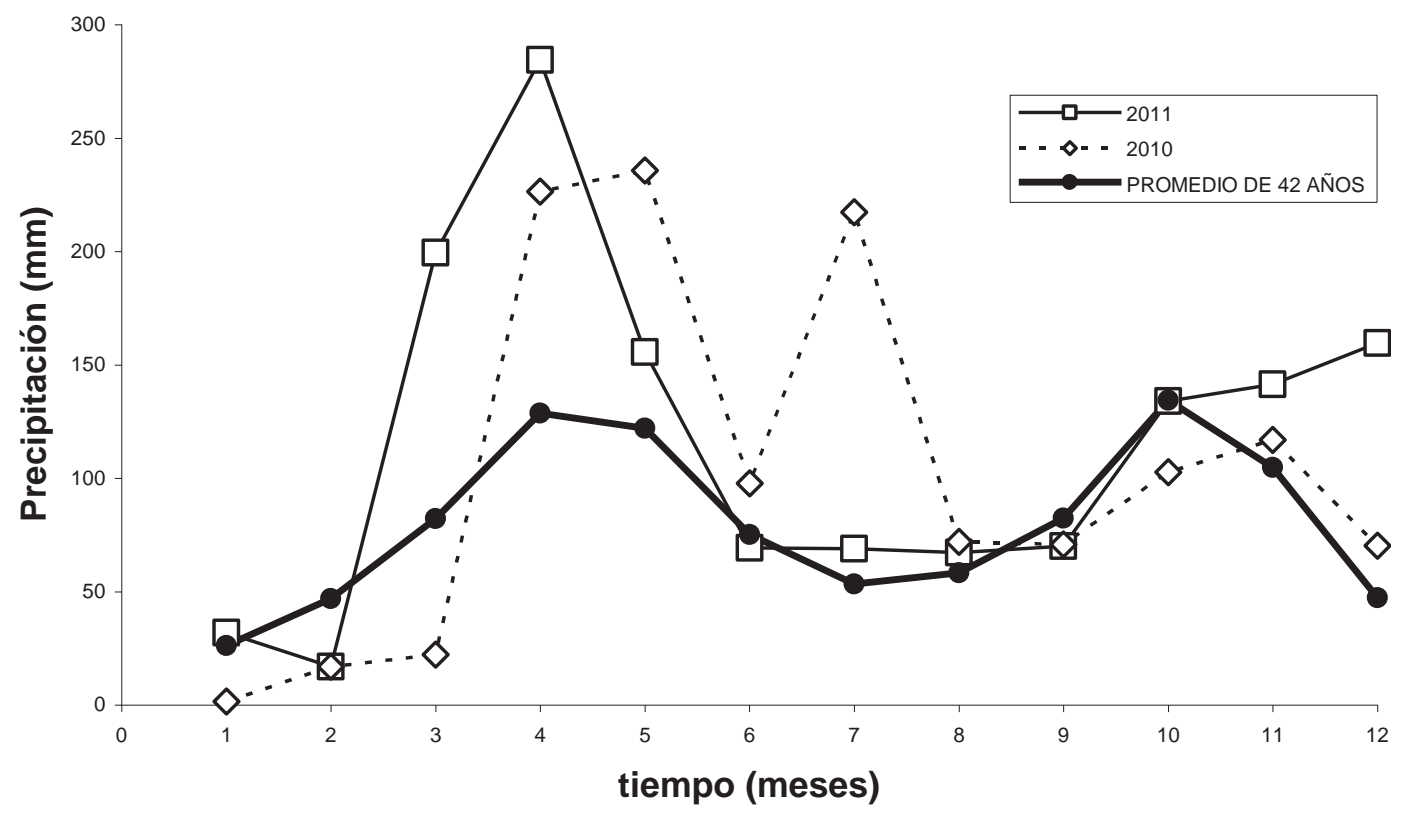

Figura 1. Precipitación medida en la estación climatológica de la granja Tunguavita (Paipa). 
variación de 30 al 45\%. Guatibonza et al. (2009) registraron CV del 57,3\%, por lo que se puede inferir, que la aireación es la relación de masa volumen con mayor CV. También se observó, que el promedio de la aireación es bajo, con un 19\%, mientras que el de la porosidad total es del $61 \%$, mostrando que la mayor parte de los poros del suelo son ocupados por el agua, lo cual, es confirmado por el porcentaje de humedad de saturación, con un promedio de $69 \%$.

Los contenidos de humedad a 4, 8, 12 y $20 \mathrm{~cm}$ tuvieron un comportamiento anormal a los $8 \mathrm{~cm}$ y normal en las otras profundidades. Este comportamiento anormal, se caracterizó por una asimetría en la distribución de los datos y un comportamiento leptocúrtico, a $4 \mathrm{~cm}$ de la superficie. Esta variabilidad en la humedad la reporta Duffera et al. (2007), quienes presentaron un coeficiente de variación, entre un 25 y 40\% y según Fang et al. (2016), de un 9 a $29 \%$. La distribución anormal de la humedad a $4 \mathrm{~cm}$ pudo ocurrir, debido a que hubo una gran concentración de datos alrededor del valor de $98 \%$, el cual, se presentó, porque el suelo, en la mayoría de los puntos, había recibido aportes de agua excesivos, producto de las máximas precipitaciones históricas en 42 años, que mostró diciembre de 2011 (Figura $1)$.

Al tomar los promedios de las distintas profundidades, se observó que el contenido de agua en el suelo disminuyó al aumentar la profundidad y pasó de valores de 94\%, a $4 \mathrm{~cm}$, a valores de $42,5 \%$, a los $20 \mathrm{~cm}$. En general, los valores de humedad a las distintas profundidades son altos, si se comparan con los resultados expuestos por Guatibonza et al. (2009), quienes hicieron el estudio en la misma zona, en época seca y obtuvieron valores que oscilaron entre 27 y $32 \%$.

La resistencia a la penetración expuso un comportamiento anormal y una distribución de los datos leptocúrtica con asimetría positiva. En las dos profundidades, 15 y $30 \mathrm{~cm}$, se observó dicha anormalidad, junto con un CV del 48 y 35\%, respectivamente, lo que resulta similar a los $\mathrm{CV}$, reportados por Duffera et al. (2007), quienes registran valores de $57 \%$ y superior a los obtenidos por Medina et al. (2012), que oscilan entre 21 y $27 \%$. Según Zhao et al. (2007), la variación de la resistencia a la penetración es afectada por el contenido de humedad en el suelo, por ello, se presentan CV diferentes, dependiendo de la zona y el contenido de humedad. Los valores promedios oscilaron entre 1,33 y $1,77 \mathrm{MPa}$, lo que indica, según Carr et al. (2013), que los datos de RP hallados se pueden considerar apropiados para la explotación agrícola; no obstante, en donde se tiene la explotación equina, debido al pisoteo de los animales, la RP superó los $4 \mathrm{MPa}$.

Análisis geoestadístico. La densidad real, humedad de saturación y humedad medida con TDR a $4 \mathrm{~cm}$ de profundidad tuvieron un GDE moderado, según la clasificación usada por Garzón et al. (2010), ya que la relación entre la meseta y el efecto pepita $[\mathrm{C} /(\mathrm{Co}+\mathrm{C})]$ es cercana a 0,5. Esta dependencia espacial, con tendencia moderada a fuerte, ha sido reportada por otros autores, como Duffera et al. (2007), quienes para humedad volumétrica, hallaron una tendencia espacial fuerte, en las capas superiores del suelo.

Es probable que esta relación de dependencia esté dada porque la humedad tiene una alta relación con la densidad aparente y la porosidad (Tabla 3). Estas variables también tienen una autocorrelación espacial de moderada a alta. Las densidades aparente y real, la porosidad, la aireación y la relación de vacíos presentan un rango superior a los $1.000 \mathrm{~m}$, es decir, que dentro de un radio de $1000 \mathrm{~m}$ estas variables son muy similares. La porosidad presentó el rango más amplio dentro de las variables evaluadas y esto sumado al bajo CV que presentó, comprueba que esta propiedad tiene una baja variabilidad (Tabla 1), por lo que si se realiza un muestreo de dichas variables, lo ideal sería realizarlas con una distancia de 1.000 metros, aproximadamente, con el fin de ahorrar recursos y así tener una medida significativa de estas propiedades del suelo.

Por otro lado, las variables de porosidad, relación de vacíos y humedad medida con TDR, a 8, 12 y $20 \mathrm{~cm}$, tienen un GDE fuerte, con valores mayores al 60\%, lo cual, se presentó, debido a que se relacionan con la Dr y Da (Tabla 2). A su vez, las densidades están muy relacionadas con el material parental y tienden a mantenerse constantes en el espacio, por lo que no cambian al aumentar la distancia de muestreo. Ejemplos de esta fuerte dependencia espacial son mostrados por Hernández et al. (2007) y Zhao et al. (2007), quienes obtuvieron un GDE del 99\%, para porosidad y del 87\%, para densidad aparente, respectivamente.

La resistencia a la penetración (RP), medida en las dos profundidades de 0 a $15 \mathrm{~cm}$ y de 15 a $30 \mathrm{~cm}$ tiene una dependencia espacial moderada, aunque el GDE varió, según la profundidad. Al respecto, Utset \& Cid (2001) exponen que la dependencia espacial de la RP cambia en relación a la humedad del suelo, con valores de GDE de 0,1 a 0,97, mientras que Kiliç et al. (2004) reportan un GDE moderado a distintas profundidades, pero dicha dependencia varía de 0,1 a 0,7 , con la profundidad y la textura del suelo.

Los rangos obtenidos para la RP estuvieron alrededor de 438 y 600m, para las dos profundidades. Estos valores contrastan con los reportados por Medina et al. (2012), quienes evaluaron RP a diferentes profundidades y encontraron rangos que oscilan entre 14 y 107m, lo cual, permite afirmar, que los datos de esta investigación tienen una mayor dependencia espacial y que las variaciones, en el nivel de compactación del suelo, son similares a lo largo 
Tabla 2. Parámetros de los semivariogramas ajustados para las variables medidas.

\begin{tabular}{|c|c|c|c|c|c|c|c|}
\hline Parámetro & Modelo & $\mathrm{C}_{0}$ & $\mathrm{C}_{0}+\mathrm{C}$ & Rango $(\mathrm{m})$ & $\begin{array}{c}\text { GDE } \\
\mathrm{C} /\left(\mathrm{C}_{0}+\mathrm{C}\right)\end{array}$ & $\mathbf{R}^{2}$ & VC \\
\hline HG (\%) & Gaussiano & 568 & 1546,9 & 788 & 0,633 & 0,699 & 0,996 \\
\hline HV (\%) & Gaussiano & 57,4 & 315,2 & 1025 & 0,818 & 0,686 & 0,710 \\
\hline HS (\%) & Exponencial & 107,5 & 271,5 & 1058 & 0,604 & 0,595 & 0,797 \\
\hline Poros (\%) & Gaussiano & 63,5 & 269,3 & 1066 & 0,764 & 0,866 & 0,723 \\
\hline A (\%) & Lineal & 45,05 & 56,93 & 496 & 0,209 & 0,606 & 0,483 \\
\hline Vacíos & Gaussiano & 0,301 & 0,897 & 1059 & 0,664 & 0,811 & 0,612 \\
\hline DA (g cm $\left.{ }^{-3}\right)$ & Gaussiano & 0,0429 & 0,2051 & 1053 & 0,791 & 0,870 & 0,811 \\
\hline DR $\left(\mathrm{g} \mathrm{cm}^{-3}\right)$ & Lineal & 0,01914 & 0,02918 & 496 & 0,344 & 0,785 & 0,692 \\
\hline H4 $(\%)$ & Lineal & 31,87 & 57,02 & 496 & 0,441 & 0,688 & 0,751 \\
\hline H8 $(\%)$ & Gaussiano & 86,1 & 323,8 & 493 & 0,734 & 0,875 & 0,963 \\
\hline H12 $(\%)$ & Gaussiano & 77 & 464,9 & 413 & 0,834 & 0,898 & 1,0 \\
\hline H20 (\%) & Gaussiano & 46,9 & 275,7 & 441 & 0,830 & 0,863 & 1,0 \\
\hline RP1 $(\mathrm{kPa})$ & Gaussiano & 2300 & 6274 & 438 & 0,500 & 0,694 & 0,141 \\
\hline RP2 $(\mathrm{kPa})$ & Gaussiano & 2540 & 8190 & 599 & 0,690 & 0,821 & 0,650 \\
\hline
\end{tabular}

Co: Efecto pepita; $\mathrm{C}_{0}+\mathrm{C}$ : meseta; $\mathrm{C} /\left(\mathrm{C}_{0}+\mathrm{C}\right)$; proporción entre la covarianza y la meseta; GDE: grado de dependencia espacial; $\mathrm{R}^{2}$ : coeficiente de ajuste del modelo del semivariograma; VC: validación cruzada. HG: humedad gravimétrica; HV: Humedad volumétrica; HS: Humedad a saturación; A: Aireación; DA: Densidad aparente en seco; DAH: densidad aparente en húmedo; DR: Densidad real; H4: humedad a 4cm por TDR; H8: humedad a 8cm por TDR; H12: humedad a 12cm por TDR; H20: humedad a $20 \mathrm{~cm}$ por TDR. RP1: resistencia a la penetración a $15 \mathrm{~cm}$; RP2: resistencia a la penetración a $30 \mathrm{~cm}$.

del transecto; no obstante, la dependencia espacial de la RP también es influenciada por características, como el contenido de materia orgánica, el tipo de sistema radicular del cultivo sembrado, la mecanización, entre otras (AlOmran et al. 2013).

Las variables de humedad medidas con TDR en todas las profundidades y la humedad de saturación tienen un rango inferior a 600m, indicando que estas variables tienen una dependencia espacial local, es decir, se mantiene una humedad similar en un radio menor a $600 \mathrm{~m}$; esto se debe, a que los contenidos de humedad dependen, principalmente, del clima y de las condiciones del suelo, por lo que se consideran que tienen una mayor variabilidad temporal que espacial (Brocca et al. 2012).

Análisis Multivariado. Se puede apreciar que la porosidad y la relación de vacíos son directamente proporcionales estadísticamente y que varían de forma inversamente proporcional con las densidades y la RP. En contraste, al observar las humedades determinadas por el TDR y la aireación tienen una relación inversamente proporcional, pero no tienen relación con la porosidad y la relación de vacíos (Tabla 3 ).
La relación entre porosidad y relación de vacíos $(r=0,95)$ fue alta, así como la relación con la densidad aparente en seco, en húmedo y real $(r=-0,97, r=-0,89, r=-0,58$, respectivamente), por lo que estas relaciones son similares a las encontradas por Guatibonza et al. (2009), en el mismo lote, pero con un muestreo en forma de malla.

La RP medida a una profundidad de 0,15 y 0,30m presentó una alta correlación negativa con la humedad volumétrica, con valores de $\mathrm{r}=-0.67$ y $\mathrm{r}=-0.54$, respectivamente; esto implica, que a mayor cantidad de agua presente en el suelo, la resistencia a la penetración será menor. En contraste, Ramírez-López et al. (2008) hallaron que la RP solo tuvo correlación con los contenidos de arena y de limo cuando fue medida a 0,10 y 0,20m; no obstante, la RP es una medida indirecta de la fuerza que ejercen las raíces, a través del suelo para crecer, absorber agua y nutrientes (Shen et al. 2016).

Los valores de humedad determinados a diferentes profundidades 4, 8, 12 y $20 \mathrm{~cm}$, tuvieron una correlación positiva con el contenido de humedad a saturación y ésta disminuyó, a medida que la profundidad de muestreo de la humedad aumentaba, por lo que se puede afirmar, que las mediciones con el TDR, a una profundidad de $4 \mathrm{~cm}$, 
Tabla 3. Valores de la correlación de Pearson entre los parámetros del suelo, evaluados en un transecto de la granja Tunguavita.

\begin{tabular}{|l|r|r|r|r|r|r|r|r|r|r|r|r|r|c|}
\hline Atributo & HG & \multicolumn{1}{|c|}{ HV } & HS & Poros & A & e & DA & DR & H4 & H8 & H12 & H20 & RP1 & RP2 \\
\hline HG & 1,00 & & & & & & & & & & & & & \\
\hline HV & 0,81 & 1,00 & & & & & & & & & & & & \\
\hline HS & 0,33 & 0,78 & 1,00 & & & & & & & & & & & \\
\hline Poros & 0,87 & 0,61 & $-0,01$ & 1,00 & & & & & & & & & & \\
\hline A & $-0,02$ & $-0,52$ & $-0,92$ & 0,37 & 1,00 & & & & & & & & & \\
\hline e & 0,89 & 0,55 & $-0,05$ & 0,96 & 0,39 & 1,00 & & & & & & & & \\
\hline DA & $-0,89$ & $-0,63$ & $-0,05$ & $-0,97$ & $-0,31$ & $-0,93$ & 1,00 & & & & & & & \\
\hline DR & $-0,69$ & $-0,45$ & $-0,13$ & $-0,59$ & $-0,11$ & $-0,56$ & 0,75 & 1,00 & & & & & & \\
\hline H4 & 0,30 & 0,47 & 0,46 & 0,13 & $-0,41$ & 0,12 & $-0,14$ & $-0,09$ & 1,00 & & & & & \\
\hline H8 & 0,17 & 0,31 & 0,34 & 0,01 & $-0,36$ & 0,02 & 0,01 & 0,05 & 0,84 & 1,00 & & & & \\
\hline H12 & $-0,08$ & 0,00 & 0,12 & $-0,22$ & $-0,23$ & $-0,16$ & 0,24 & 0,20 & 0,61 & 0,87 & 1,00 & & & \\
\hline H20 & $-0,08$ & $-0,04$ & 0,09 & $-0,25$ & $-0,22$ & $-0,17$ & 0,26 & 0,17 & 0,59 & 0,85 & 0,97 & 1,00 & & \\
\hline RP1 & $-0,47$ & $-0,67$ & $-0,52$ & $-0,46$ & 0,30 & $-0,35$ & 0,45 & 0,22 & $-0,39$ & $-0,30$ & $-0,04$ & 0,04 & 1,00 & \\
\hline RP2 & $-0,39$ & $-0,55$ & $-0,37$ & $-0,44$ & 0,16 & $-0,34$ & 0,44 & 0,19 & $-0,29$ & $-0,19$ & 0,04 & 0,14 & 0,84 & 1,00 \\
\hline
\end{tabular}

HG: humedad gravimétrica; HV: Humedad volumétrica; HS: Humedad a saturación; A: Aireación; e: relación de vacíos; DA: Densidad aparente en seco; DAH: densidad aparente en húmedo; DR: Densidad real; H4: humedad a 4cm por TDR; H8: humedad a $8 \mathrm{~cm}$ por TDR; H12: humedad a 12cm por TDR; H20: humedad a 20cm por TDR. RP1: resistencia a la penetración a $15 \mathrm{~cm}$; RP2: resistencia a la penetración a $30 \mathrm{~cm}$.

tienen muy buena correlación con la humedad a saturación, situación que se pudo dar, debido a los fuertes eventos de precipitación con máximos históricos ocurridos, lo cual, en general, hizo que todos los datos de humedad presentaran valores altos y que la mayoría de los poros del suelo, tuvieran grandes contenidos de agua, lo que explica, además, la relación inversamente proporcional entre la aireación y los contenidos de humedad a saturación y a diferentes profundidades medidos con el TDR.

Componentes principales (CP). Se obtuvieron tres $\mathrm{CP}$, los cuales, sumados, explicaron en un $85,47 \%$ la varianza total de los datos, valor superior al $80 \%$, que es el valor requerido para realizar un buen análisis estadístico (Medina et al. 2012). En la tabla 4, se aprecia la influencia de las variables medidas dentro del primer componente, que explica la varianza total de los datos en un 41,4\%. En el CP1, la variable que presentó mayor influencia fue la porosidad, seguida de la humedad gravimétrica, de la relación de vacíos y de la humedad volumétrica. Lo anterior indica que el suelo muestreado esta dominado por la influencia del régimen de humedad del suelo y la relación de la fase líquida con la fase gaseosa del suelo.

El segundo CP representó un 28,9\% y estuvo afectado, principalmente, por las humedades medidas en el TDR, a diferentes profundidades y la humedad a saturación, que mostraron una alta correlación positiva entre sí. Nuevamente, el contenido de humedad presentado en el suelo, se mostró como el principal factor que afectó los fenómenos que determinan la capacidad del suelo, para su uso agrícola. El tercer CP tuvo un efecto del $15 \%$ sobre la varianza de los datos, afectado, principalmente, por la humedad medida con TDR, a los 20 y 15cm y la aireación, situación que revela el efecto de las altas precipitaciones, que mantienen una alta humedad en el suelo en profundidad.

Al comparar el CP1 y el CP2, muestran dos grandes grupos de variables que se relacionan entre sí. En el grupo 1, se encuentran las humedades, las densidades y las relaciones de masa volumen y, en el grupo 2, las RP, a 15 y $30 \mathrm{~cm}$. La aireación presentó poco efecto sobre las demás variables y se encuentra alejada de los grupos de variables.

La densidad aparente fue la variable que presentó el mayor valor de comunalidad, lo que implica que es la propiedad que más influye y, a su vez, que es la más afín a los demás atributos medidos; esto ocurre, debido a que la densidad aparente desempeña un papel importante en el cálculo de las relaciones de masa-volumen, así como también sobre la RP. Al respecto, Hakansson \& Lipiec (2000) mencionan que el grado de compactación del suelo tiene una gran correlación 
Tabla 4. Coeficientes de los componentes principales para los parámetros evaluados.

\begin{tabular}{|c|c|c|c|c|}
\hline Parámetro & CP1 & CP2 & CP3 & Comunalidad \\
\hline HG (\%) & 0,933 & 0,119 & 0,045 & 0,885 \\
\hline HV (\%) & 0,761 & 0,490 & $-0,337$ & 0,820 \\
\hline HS (\%) & 0,232 & 0,720 & $-0,594$ & 0,572 \\
\hline Poros (\%) & 0,949 & $-0,172$ & 0,165 & 0,931 \\
\hline A (\%) & 0,131 & $-0,761$ & 0,573 & 0,596 \\
\hline Vacíos & 0,905 & $-0,178$ & 0,250 & 0,851 \\
\hline DA ( cm $\left.^{-3}\right)$ & $-0,972$ & 0,164 & $-0,124$ & 0,972 \\
\hline DR ( cm$\left.^{-3}\right)$ & $-0,714$ & 0,120 & $-0,052$ & 0,524 \\
\hline H4 (\%) & 0,244 & 0,808 & 0,265 & 0,712 \\
\hline H8 (\%) & 0,075 & 0,857 & 0,465 & 0,739 \\
\hline H12 (\%) & $-0,202$ & 0,730 & 0,605 & 0,573 \\
\hline H20 (\%) & $-0,233$ & 0,696 & 0,637 & 0,539 \\
\hline RP1 (kPa) & $-0,605$ & $-0,439$ & 0,311 & 0,559 \\
\hline RP2 (kPa) & $-0,570$ & $-0,300$ & 0,300 & 0,415 \\
\hline Autovalor & 6,21 & 4,34 & 2,25 & \\
\hline Varianza total \% & 41,45 & 28,97 & 15,05 & \\
\hline Varianza acumulada & 41,45 & 70,42 & 85,47 & \\
\hline
\end{tabular}

CP: Componente principal.

con los valores de la RP, ya que, según Lipiec \& Hatano (2003), la densidad aparente en relación con la densidad aparente obtenida por compresión uniaxial a una presión de $200 \mathrm{kPa}$, es definida como el grado de compactación del suelo.

Asimismo, la porosidad presentó el segundo valor más alto de comunalidad, lo que implica que esta variable, fuertemente ligada a la densidad real, también esta relacionada, tanto con los contenidos de humedad, como con las densidades y la RP. En tercer lugar, aparece la humedad gravimétrica, seguida, de la relación de vacíos, los cuales, poseen altos valores de comunalidad, debido a que son atributos afectados por la densidad aparente. En cuanto, a los valores de comunalidad de las humedades obtenidas a partir del TDR, se puede decir, que a mayor profundidad, el valor de comunalidad de la humedad va disminuyendo, porque disminuye el efecto que esta pueda tener sobre las propiedades físicas medidas en el primer horizonte del suelo.

Conflictos de intereses: El manuscrito fue preparado y revisado con la participación de todos los autores, quienes declaran que no existe conflicto de intereses que ponga en riesgo la validez de los resultados presentados.

\section{BIBLIOGRAFÍA}

1. ALLAIRE, S.E.; LANGE, S.F.; LAFOND, J.A.; PELLETIER, B.; CAMBOURIS, A.N.; DUTILLEUL, P. 2012. Multiscale spatial variability of $\mathrm{CO}_{2}$ emissions and correlations with physico-chemical soil properties. Geoderma 170:251-260.

2. AL-OMRAN, A.M.; AL-WABEL, M.I.; EL-MAGHRABY, S.E.; NADEEM, M.E.; AL-SHARANI, S. 2013. Spatial variability for some properties of the wastewater irrigated soils. J. Saudi Soc. Agr. Sci. 12:167-175.

3. BROCCA, L.; TULLO, T.; MELONE, F.; MORAMARCO, T.; MORBIDELLI, R. 2012. Catchment scale soil moisture spatial-temporal variability. J. Hydrology. 422-423:63-75.

4. CARR, P.; GRAMIG, G.; LIEBIG, M. 2013. Impacts of organic zero tillage systems on crops, weeds, and soil quality. Sustainability 5(7):3172-3201.

5. CUCUNUBA-MELO, J.; ALVAREZ-HERRERA, J., CAMACHO TAMAYO, J. 2011. Identification of agronomic managment units based on physical 
attibutes of soil. J. Soil Sci. Plant Nutrition. 11(1):8799.

6. DOS SANTOS, D.; DE SOUZA, E.G.,; NÓBREGA, L.H.; BAZZI, C.L.; GONÇALVES, A.C. 2012. Variabilidade espacial de atributos físicos de um Latossolo Vermelho após cultivo de soja. Rev. Bras. Engenh. Agr. Amb. 16(8):843-848.

7. DUFFERA, M.; WHITE, J.G.; WEISZ, R. 2007. Spatial variability of Southeastern U.S. Coastal Plain soil physical properties: Implications for site-specific management. Geoderma. 137(3-4):327-339.

8. FANG, K.; LI, H.; WANG, Z.; DU, Y.; WANG, J. 2016. Comparative analysis on spatial variability of soil moisture under different land use types in orchard. Scientia Horticulturae. 207:65-72.

9. GARZÓN, C.A.; CORTÉS, C.A.; CAMACHO-TAMAYO, J.H. 2010. Variabilidad espacial de algunas propiedades químicas en un Entisol. Rev. U.D.C.A. Act. \& Div. Cient. 13(1):87-95.

10. GLENDELL, M.; GRANGER, S.J.; BOL, R.; BRAZIER, R.E. 2014. Quantifying the spatial variability of soil physical and chemical properties in relation to mitigation of diffuse water pollution. Geoderma. 214-215:25-41.

11. GUATIBONZA, M.; ÁLVAREZ-HERRERA, J.; SANABRIA, J. 2009. Distribución espacial de la conductividad hidráulica en un lote de la granja Tunguavita (Paipa, Colombia). Agr. Col. 27(2):261-271.

12. HAKANSSON, I.; LIPIEC, J. 2000. A review of the usefulness of relative bulk density values in studies of soil structure and compaction. Soil Tillage Res. 53:71-85.

13. HERNÁNDEZ, P.; FERNÁNDEZ, R.; NOVO, M.; TRIGO, D.; DÍAZ, D.J. 2007. Geostatistical and multivariate analysis of the horizontal distribution of an earthworm community in El Molar (Madrid, Spain). Pedobiologia. 51(1):13-21.

14. IQBAL, J.; THOMASSON, A.; JENKINS, J. N.; OWENS, P. R.; WHISLER, F.D. 2005. Spatial variability analysis of soil physical properties of alluvial soils. Soil Sci. Soc. Am. J. 69:1338-1350.

15. KILIÇ, K.; ÖZGÖZ, E.; AKBAŞ, F. 2004 Assessment of spatial variability in penetration resistance as related to some soil physical properties of two fluvents in Turkey. Soil Tillage Res. 76(1):1-11.

16. LIPIEC, J.; HATANO, R. 2003. Quantification of compaction effects on soil physical properties and crop growth. Geoderma. 116:107-136.

17. MEDINA, C.; CAMACHO-TAMAYO, J.; CORTÉS, C. 2012. Soil penetration resistance analysis by multivariate and geostatistical methods. Eng. Agric. 32(1):91101.

18. MULLA, D.J. 2012. Chapter 20 - Modeling and Mapping Soil Spatial and Temporal Variability. En: LIN, H. (ed). Hydropedology. Academic Press (Boston). p.637664.

19. RAMÍREZ-LÓPEZ, L.; REINA-SÁNCHEZ, A.; CAMACHOTAMAYO, J.H. 2008. Variabilidad espacial de atributos físicos de un Typic Haplustox de los Llanos Orientales de Colombia. Eng. Agric. 28(1):55-63.

20. SHEN, Q.; GAO, G.; HU, W.; FU, B. 2016. Spatialtemporal variability of soil water content in a croplandshelterbelt-desert site in an arid inland river basin of Northwest China. J. Hydrology. 540:873885.

21. SUN, B.; ZHOU, S.; ZHAO, Q. 2003. Evaluation of spatial and temporal changes of soil quality based on geoestatistical analysis in the hill region of subtropical China. Geoderma. 115(1-2):85-99.

22. TASUMI, M.; KIMURA, R. 2013. Estimation of volumetric soil water content over the Liudaogou river basin of the Loess Plateau using the SWEST method with spatial and temporal variability. Agr. Water Managem. 118:22-28.

23. USDA. 2004. Soil Survey Laboratory. Methods Manual. Investigation report No. 42. Versión 4.0. 700p. United States Department of Agriculture, Washington D.C., USA.

24. UTSET, A.; CID, G. 2001. Soil penetrometer resistance spatial variability in a Ferralsol at several soil moisture conditions. Soil Tillage Res. 61(3-4):193-202.

25. ZHAO, Y.; PETH, S.; KRÜMMELBEIN, J.; HORN, R.; WANG, Z.; STEFFENS, M. 2007. Spatial variability of soil properties affected by grazing intensity in Inner Mongolia grassland. Ecol. Modelling 205(1-2):241254. 
26. ZUCCO, G.; BROCCA, L.; MORAMARCO, T.; Recibido: Agosto 19 de 2016 MORBIDELLI R. 2014. Influence of land use on soil Aceptado: Febrero 7 de 2017 moisture spatial-temporal variability and monitoring. J. Hydrology 516:193-199.

\section{Cómo citar.}

Monroy-Rodríguez, F.L.; Álvarez-Herrera, J.G.; Alvarado-Sanabria, O.H. 2017. Distribución espacial de algunas propiedades físicas del suelo en un transecto de la granja Tunguavita, Paipa. Rev. U.D.C.A Act. \& Div. Cient. 20(1): 91-100. 